\title{
Discharge Planning for Palliative Care Patients: A Qualitative Analysis
}

\author{
Emma Benzar, B.S., ${ }^{1}$ Lissi Hansen, R.N., Ph.D., ${ }^{2}$ Anna W. Kneitel, M.D., ${ }^{3}$ Erik K. Fromme, M.D. ${ }^{4}$
}

\begin{abstract}
For patients hospitalized with life-threatening illnesses and their families, palliative care consultants can provide critical support by providing information about prognosis, ensuring that symptoms are managed, helping to clarify goals of care, and addressing psychosocial and spiritual concerns. However, once patients leave the hospital, many hospital-based palliative care teams (PCTs) cannot continue to play active roles in patient care. Gaps in discharge planning not only decrease quality of life for patients, but also translate into lack of support for caregivers. The palliative care population would be expected to benefit from a customized approach to hospital discharge. The aim of this study was to identify the range of health care experiences of family caregivers and patients who received palliative care consultations after they left the hospital, and to understand how PCTs might best prepare patients and caregivers for the post-hospital experience.
\end{abstract}

\section{Introduction}

$\mathbf{D}^{\prime}$ ISCHARGE PLANNING is a core element of hospital-based palliative care consultation. ${ }^{1}$ For patients hospitalized with life-threatening illnesses and their families, palliative care consultants can provide critical support by providing information about prognosis, ensuring that symptoms are managed, helping to clarify goals of care, and addressing psychosocial and spiritual concerns. However, once patients leave the hospital, many hospital-based palliative care teams (PCTs) do not have the structure or resources to allow them to continue to play an active role in patients' care. ${ }^{2}$ This creates a "care transition" from the hospital to the next setting of care, which could be a private home or care facility. Multiple transitions between care settings are common in the last six months of life, and are associated with family reports of too much or too little life-sustaining care and barriers to carrying out patient preferences. ${ }^{3}$

Numerous studies have identified flaws in discharge planning for general patient populations. For instance, Moore and colleagues found that $49 \%$ of patients seen in clinic after hospital discharge had problems related to medications, test follow-up, or completion of a planned work-up. ${ }^{4}$ Of these, medication discrepancies were the most frequent concerns $(42 \%)$. These gaps in discharge planning not only decrease quality of life for patients, but also translate into lack of support for caregivers. 5

However, no systematic work has been done to identify the perspectives of patients and caregivers on discharge from U.S. hospitals following palliative care consultation. A previous study at Oregon Health and Science University (OHSU) found that, following palliative care consultation, one-third of patients died in the hospital and two-thirds of patients were discharged alive. Of patients discharged alive, only $10 \%$ were readmitted to OHSU within 30 days, $50 \%$ died within 12 days of discharge, and only $5 \%$ ultimately died in acute care hospitals. ${ }^{7}$ In this study, many palliative care patients were exceedingly ill at the time of hospital discharge. The high rate of death outside the hospital raises important questions: were patients adequately prepared for discharge, and did they receive good care in their new settings?

Families and caregivers of palliative care patients face added burdens-in addition to managing complex care needs, they must also navigate home health systems, make legal arrangements, and cope with grief. ${ }^{8}$ In light of these complex needs, the palliative care population would be expected to benefit from a customized approach to hospital discharge.

The aim of this study was to identify the range of health care experiences of family caregivers and patients who received palliative care consultations at OHSU after they left the

\footnotetext{
${ }^{1}$ Oregon Health \& Science University, School of Medicine, Portland, Oregon.

${ }^{2}$ Oregon Health \& Science University, School of Nursing, Portland, Oregon.

${ }^{3}$ Department of Obstetrics and Gynecology, University of California, Davis, Davis, California.

${ }^{4}$ Division of Hematology and Medical Oncology, Oregon Health \& Science University, Portland, Oregon.

Accepted August 3, 2010.
} 
hospital. Another aim was to understand how PCTs might best prepare patients and caregivers for the post-hospital experience.

\section{Methods}

This was a retrospective, qualitative study of patients who received palliative care consults at OHSU. The Institutional Review Board at OHSU approved this study and all subjects gave informed consent.

OHSU is a tertiary care academic hospital with 509 staffed adult beds. In 2006, the Palliative Medicine and Comfort Care Team consisted of two physicians and a clinical nurse specialist who work closely with hospital case managers, social workers, and chaplains employed on each hospital unit. The team operates as a consult service. Approximately 33\% of consults come from the general medicine or family practice wards, $21 \%$ from critical care and cardiology, and $13 \%$ from hematology/medical oncology and bone marrow transplantation. ${ }^{9}$

The study was based on in-depth interviews with 19 patients and/or their caregivers. Palliative care team documentation in the medical chart was also reviewed to characterize the consultations. Subjects were drawn from 494 consecutive patients who had received palliative care consults at OHSU from September 1, 2006-August 31, 2007. The inclusion criteria were patients who were: (1) discharged alive between two weeks and three months before identification of patient for interview, (2) discharged to either home without hospice, home with hospice, nursing home, or inpatient hospice, and (3) English-speaking. Subjects were purposively recruited to ensure inclusion of subjects who were non-white, did not carry a diagnosis of cancer, and were discharged to nursing homes. Purposive sampling is an accepted technique in qualitative research to ensure that the appropriate range of experiences and viewpoints is included in the sampling frame. ${ }^{10}$

Potential subjects were contacted by phone after receiving an introductory letter and consent form by mail. A family or friend caregiver who was self-identified as "very involved" in care and decision making for the patient was also invited to participate. Patients and/or caregivers participated in one- to two-hour, semi-structured interviews based on a scripted questionnaire. The questionnaire covered caregiver and patient demographics, experiences with care after hospital discharge, and the quality of the discharge process as preparation for this setting. Of 19 interviews, 11 were in person and 8 were over the phone; 15 interviews were with caregivers only, 1 was with the patient alone, and 3 were with both.

Interviews were analyzed by two independent coders using NVivo v.8, a qualitative data-analysis program (QSR International, Cambridge, MA). This study started with broad questions that allowed subjects to determine, in the context of semi-structured interviews, what was important about their experience with palliative care and the discharge process. The interviews were then analyzed using qualitative descriptiona method of choice when straightforward and less-interpreted answers to questions are desired. ${ }^{11}$ Based on the strategy described, a researcher coded all 19 interview transcripts line by line, identifying 84 discrete codes. Using a pile sort, these codes were then organized into 15 codes within 6 major themes. Based on these themes, the interview transcripts were re-coded by the original researcher and independently by a second researcher. Intercoder reliability was calculated as letter-by-letter agreement using NVivo. Of the 6 themes, 3 were concerns that could potentially be addressed by a PCT before hospital discharge. We chose to focus our further analysis on these.

The other three themes were related to experiences after hospital discharge that were outside of the scope of an inpatient palliative care team and, thus, outside the scope of this study. Our data is presented in the form of quotes that represent the three major themes of concern most relevant to discharge planning for palliative care patients.

\section{Results \\ Subject characteristics}

Subject characteristics are outlined in Table 1. Ethnic minorities represented by patients included black/AfricanAmerican $(\mathrm{n}=2)$, American Indian (1), and Hispanic/Latino (1). Ethnic minorities represented by caregivers included black/African-American $(n=2)$, American Indian (2), and Hispanic/Latino (1). Six patients carried a diagnosis of cancer. Other diagnoses included abdominal abscess $(n=2)$, mastocytosis, urosepsis, aortic stenosis, gastrointestinal bleed, hepatitis C-related liver failure, hepatic encephalopathy, renal failure, chronic obstructive pulmonary disease, small bowel obstruction, failure to thrive, and implantable cardioverter defibrillator wire complication. Similar to the patients in our previous study, $52 \%$ of patients in this study cohort died within 12 days of discharge. Median survival was 11 days.

\section{Consult characteristics}

According to chart documentation, the median length of initial consult was 81 minutes (range $45-120$ minutes) and the median number of interactions was visits was 2 (range 1-5). Discussions about goals of care were documented in 11/19 (58\%) visits, symptoms or quality of life in 14/19 (74\%), prognosis in $4 / 19(21 \%)$, and discharge location or hospice in $12 / 19$ (63\%). Most consults mentioned more than one emphasis. Common symptoms discussed included pain, nausea, depressed mood, and weakness.

\section{Interview themes}

We identified three themes of concern that could be addressed by palliative care teams during discharge planning. Not all patients identified these as problems, but each theme emerged from at least a few patients. Inter-coder reliability was excellent, with a letter-by-letter agreement of $96 \%$.

Theme 1: Prognosis. Lacking information about prognosis and disease progression was a recurrent theme. In the hospital, prognoses were sometimes vague or over-optimistic and lacked information about what caregivers should expect.

The first quote demonstrating this concern is from the daughter of a 58-year-old female, discharged with mastocytosis and ascites to inpatient hospice. The main documented goal of this palliative care consult was to discuss goals of care and prognosis, and the patient's chart confirmed that these 
topics had been discussed. However, the patient's daughter described doctors' attempts to discuss prognosis as:

"The biggest dance around' you're going to die I've ever heard," and,

"The hardest thing was that I had to tell her instead of [her] really

hearing it from the medical professionals." [Subject \#10]

The son of an 85-year-old male with bladder cancer, discharged to home hospice, expressed concern that he had to read into what the doctors were saying about his father's impending death. He was concerned that not everyone would have been able to draw the conclusion that his father was about to die. He noted:

\section{"It was all my reading into it that, when the doctor said hospice, that meant [my father] was going... And there was always a little lin- gering doubt about what time frame are we talking about ... It could have been ... useful just to say, 'You need to prepare for his passing.'" [Subject \#32]}

The daughter of a 56-year-old female who originally went home with hospice for cancer was frustrated when her mother died unexpectedly awhile later from a dialysis shunt infection. The primary team involved palliative care only when tumor resection was considered impossible. However, the patient's daughter was still surprised at her mother's death and said:

"I never knew to watch for signs for a shunt to have an infection ... For the three or four months that we have been knowing about this cancer, they've been telling us all along, that, 'no problem, we're going to do surgery.' We've got nothing but good news. So when I got the call that 'that's it, that it's over,' I was in shock, total shock, I couldn't believe it." [Subject \#7]

Theme 2: Symptom management. The second major theme that arose was that of symptom management. We found that families often lacked the education that they needed to recognize and manage symptoms. In some cases, teaching about medicine use for symptoms was sparse and not written down. If dosing instructions on the prescription bottle were inadequate, caregivers had no contingency plans.

In the words of a caregiver whose 59-year-old mother, who had renal cell carcinoma, went home without hospice:

"They prescribed her over $\$ 1000$ in medicines... The discharge paper was blank... They didn't give her what she needed. They changed what was working to other medicines. It seemed like we had never spoken to those people at all..." [Subject \#5]

When the story was verified, the discharge medication summary had indeed been blank, and the dosing instructions provided by the pharmacy did not correspond with the doses the patient had been taking in the hospital.

The caregiver of a 55-year-old male on home hospice for end-stage liver disease was not prepared to adjust medication doses when needed. Although symptom control was a major emphasis of this patient's palliative care consult in the hospital, after hospital discharge, the caregiver said:

"We were kind of confused on the pain medication... We were giving what they said he should have and I was leaving him sleeping all day

long... And he didn't want to be that way and I personally didn't want to see him that way either." [Subject \#30]
Theme 3: Whom to call with questions. A third area of concern was whom to contact with questions and concerns. Some quotes also reflect difficulty with simply navigating the healthcare system. Interestingly, both of the patients mentioned had only one documented encounter with palliative care during their stay.

The daughter of an 85-year-old female who went home without hospice after a GI bleed had difficulty learning about options for home care. She said,

"Several times we didn't know what was out there to help us... She wanted to come home and we wanted to provide care for her at home... But we didn't know what other type of care there was ... We finally got some of that information but... We had to struggle to search it out, and answers weren't readily available." [Subject \#23]

The daughter of a 71-year-old female with metastatic cancer on inpatient hospice described how grateful she was to find a receptionist at the doctor's office to help her through the process. She commented:

\section{"The medical system, if you are not part of it, is a pretty foreign thing... If you find a person that will work with you, whether it be a doctor, a nurse... the scheduler... They are there to help guide you through the system. But finding them and really cultivating that relationship makes a huge difference." [Subject \#16]}

Hospice. Only one of the preceding quotations came from a patient cared for by a hospice program. Our data included multiple examples in which patients and families with hospice mentioned that hospice nurses answered all their questions, helped them adjust medications, and contacted the physicians for them. However, only $53 \%$ of patients in this study were discharged to home or inpatient hospice (Table 1). Additionally, even patients discharged with hospice experienced gaps in the discharge planning process before they left the hospital.

Palliative care teams. Another key finding was that, at the time of their interviews (up to three months after hospital discharge), several patients and their families did not remember their interactions with the PCT, despite being shown pictures of team members to help jog their memories. In other cases, interviewees remembered the PCT members but could not distinguish interactions with them from interactions with other types of care teams.

\section{Discussion}

This is the first U.S. study to undertake a systematic review of the discharge planning process in palliative care, particularly from the perspective of patients and caregivers. In retrospect, the first insight from this process is obvious - that the transition from the hospital to another care setting is a complex experience involving a huge number of variables, as illustrated by our initial attempt at coding resulting in 84 codes. Even after limiting ourselves to 15 codes, we found ourselves needing to focus on the three areas of concern that could be anticipated and addressed by the PCT before discharge.

The themes that we identified were prognosis, symptom management, and knowing whom to call when problems arose. Deficits in these areas are potentially preventable and can be addressed before a patient leaves the hospital. However, they require complex interdisciplinary participation, 
Table 1. Patient and Caregiver Characteristics

\begin{tabular}{|c|c|c|}
\hline & $\mathrm{n}($ total $=19)$ & $\%$ \\
\hline \multicolumn{3}{|l|}{ Patient characteristics } \\
\hline Mean age (SD) & 66 years $(16)$ & $\mathrm{n} / \mathrm{a}$ \\
\hline Female & 11 & 58 \\
\hline Ethnic minorities & 4 & 21 \\
\hline Alive for interview & 4 & 21 \\
\hline \multicolumn{3}{|c|}{ Pt location of care after hospital discharge } \\
\hline Inpatient hospice & 4 & 21 \\
\hline Home hospice & 6 & 32 \\
\hline Home, no hospice & 7 & 37 \\
\hline Nursing home & 2 & 10 \\
\hline \multicolumn{3}{|c|}{ Survival after hospital discharge } \\
\hline$\leq 5$ days & 5 & 26 \\
\hline $6-12$ days & 5 & 26 \\
\hline $13-30$ days & 5 & 26 \\
\hline 31-90 days & 0 & 0 \\
\hline \multirow[t]{2}{*}{$>90$ days } & 4 & 21 \\
\hline & $\mathrm{n}($ total $=19)$ & $\%$ \\
\hline \multicolumn{3}{|l|}{ Caregiver characteristics } \\
\hline Mean age (SD) & 50 years $(13)$ & $\mathrm{n} / \mathrm{a}$ \\
\hline Female & 14 & 74 \\
\hline Ethnic minorities & 5 & 26 \\
\hline \multicolumn{3}{|c|}{ Caregiver relationship to patient } \\
\hline Daughter/stepdaughter & 8 & 42 \\
\hline Wife & 2 & 11 \\
\hline Son/stepson & 2 & 11 \\
\hline Friend & 2 & 11 \\
\hline Other & 5 & 26 \\
\hline
\end{tabular}

so responsibility for addressing them is often ambiguous. Based on the findings of this study, we suggest that PCTs could take responsibility for these three areas. This suggestion is supported by the National Consensus Project, which recently included these themes as preferred practices in its report of quality indicators in palliative care. ${ }^{12}$

Studies have shown that many families want to be informed of prognosis and disease progression so that they can prepare emotionally and logistically for a loved one's death. ${ }^{13}$ Even so, physicians can be reluctant to discuss this, fearing that they may take away hope. ${ }^{14}$ Although the OHSU PCT discussed goals of care or hospice with most patients, our study identified instances in which these discussions did not include an explicit-enough discussion of prognosis. We suggest that PCTs ask how much families already know, how much they want to know, and when they are ready to hear more. ${ }^{15}$

Like prognosis, symptom management is a major focus of the OHSU PCT $-74 \%$ of its time, in the current study. Although achieving optimal symptom control is challenging in the hospital, ensuring that the patient will be comfortable after hospital discharge, often in the care of untrained family members, is even more challenging. ${ }^{16,17}$ Our study suggests that PCTs have to address this transitional gap. However, there are several systemic barriers. At OHSU, the PCT does not write the final discharge summary. Additionally, past interventions aimed at discharge summaries have not always led to statistically significant improvements. ${ }^{18}$ This may be because patients frequently face psychosocial problems that are not anticipated by discharge summaries, or because much of the planning for discharge is lost in transition. ${ }^{19,20}$

Because of these potential problems, patients at OHSU are usually told to call the hospital if they have questions after discharge. Unfortunately, at a large hospital, whom to call is not always clear. The discharge summary contains a standardized phone number, but individual health care providers or social workers often give families personal contact information as well. As evident in our quotes, this can leave caregivers confused or overwhelmed. We propose that PCTs identify one individualized number for patients and caregivers to call. The person designated to answer should be familiar with the patient's history and should be able to find answers quickly.

As the National Consensus Project suggests, addressing these themes of concern before hospital discharge is vital to quality care for palliative care patients. However, there is no substitute for actual follow-up after discharge, as evident in the ability of programs such as hospice to make up for gaps in the transition to home. For instance, in a study by Teno et al., the percent of patients and families complaining of inadequate pain support was $43 \%$ in home health, $32 \%$ in nursing homes, and only $18 \%$ in hospice. ${ }^{21}$ However, not all palliative care patients qualify for hospice. Additionally, patients at regional hospitals like OHSU may lack insurance or lack access to local care. For these reasons, groups around the nation are experimenting with interventions to follow specific populations of patients regularly after they leave the hospital. Some of these programs have already demonstrated lower hospital charges and re-hospitalization rates. ${ }^{22,23,24,25}$ Our study highlights the potential importance of providing palliative care services in the outpatient setting for patients not enrolled in hospice.

This study has several limitations. Because of the in-depth interview analysis necessary for a qualitative study, we were only able to represent 19 of 494 patients seen during one year. However, our demographic data, as shown in the table, suggests that our purposive sampling method for subject selection was effective in including a variety of experiences. Additionally, the open-ended interviewing technique allowed subjects to raise a variety of concerns, although it was not generally possible to distinguish which problems could be attributed to the palliative care team. Also, because we decided to focus on concerns that could possibly be acted on by palliative care teams and not all subjects had these problems, this analysis does not represent all subjects' experiences equally. It also includes the authors' potential biases about what "could possibly be acted on by palliative care teams."

Our reliance on self-report was also a limitation. Since our median palliative care patient survival is only 11 days, most interviews were with caregivers rather than patients themselves. Obtaining information while memories were fresh was desirable, but patients were often too ill to provide much information and caregivers' responses could have been influenced by their recent losses.

This study also has several strengths. The qualitative methods allowed us to identify concerns we had not anticipated, such as the degree to which several subjects could not recall their interactions with the PCT. The ability to ask follow-up questions in the in-depth interviews allowed us to better understand the reasons behind some of the identified problems. Interviewing caregivers and patients discharged to 
multiple settings, with and without hospice care, helped us understand the different problems that arise depending on where the patient goes.

\section{Conclusion}

In conclusion, we identified three of the quality indicators for palliative care that were important to patients and caregivers. Our study suggests that understanding prognosis, being prepared to manage symptoms, and knowing whom to call with questions would help families cope more effectively with the challenges of life-threatening illness. Although systemic barriers make it difficult to assign responsibility for these issues, we suggest that PCTs nationwide are uniquely suited to initiate change. In doing so, palliative care professionals would be making significant strides to improve the quality of care for both patients and their loved ones.

\section{Acknowledgments}

An abstract and oral presentation of this work was presented at the 2009 American Academy of Hospice and Palliative Medicine Annual Assembly in Austin, Texas.

The authors thank all the study participants, who so generously contributed their stories to make this study possible. Special thanks to Susan W. Tolle, M.D., Tawni KenworthyHeinige, B.S., CCRP, Paul B. Bascom, M.D., Mary Denise Smith, R.N., M.S., C.N.S.

Dr. Fromme is supported by a Career Development Award from the National Cancer Institute K07CA109511. This publication was also made possible with support from the Oregon Clinical and Translational Research Institute (OCTRI), grant number UL1 RR024140 and TL1 RR024159 from the National Center for Research Resources (NCRR), a component of the National Institutes of Health (NIH), and NIH Roadmap for Medical Research.

\section{Author Disclosure Statement}

No competing financial interests exist.

\section{References}

1. Weissman DE: Consultation in palliative medicine. Arch Intern Med 1997;157:733-737.

2. Meier DE, Beresford L: Palliative care's challenge: facilitating transitions of care. J Palliat Med 2008;11:416-421.

3. Tolle SW, Tilden VP, Rosenfeld AG, Hickman SE: Family reports of barriers to optimal care of the dying. Nurs Res 2000;49:310-317.

4. Moore C, Wisnivesky J, Williams S, McGinn T: Medical errors related to discontinuity of care from an inpatient to an outpatient setting. J Gen Intern Med 2003;18:646-651.

5. Grimmer KA, Moss JR, Gill TK: Discharge planning quality from the career perspective. Qual Life Res 2000;9:1005-1013.

6. Levine C: The loneliness of the long-term care giver. NEJM 1999;340:1587-1590.

7. Fromme EK, Bascom PB, Smith MD, Tolle SW, Hanson L, Hickam DH, Osborne ML: Survival, mortality, and location of death for patients seen by a hospital-based palliative care team. J Palliat Med 2006;9:903-911.

8. Apatira L, Boyd EA, Malvar G, Evans LR, Luce JM, Lo B, White DB: Hope, truth, and preparing for death: perspectives of surrogate decision makers. Ann Intern Med 2008; 149:861-868.
9. Fromme EK, Bascom PB, Smith MD, Tolle SW, Hanson L, Hickam DH, Osborne ML: Survival, mortality, and location of death for patients seen by a hospital-based palliative care team. J Palliat Med 2006;9:903-911.

10. Bernard HR: Social research methods: qualitative and quantitative approaches. Thousand Oaks, CA: Sage Publications, 2000.

11. Sandelowski M: Whatever happened to qualitative description? Res Nurs Health 2000; 23:334-40.

12. National Consensus Project: Clinical practice guidelines for quality palliative care: Domains 1, 2, and 7. 2nd ed. National Consensus Project for Quality Palliative Care 2009. Accessed 13 February 2010 at http://www.nationalconsensusproject. org/guideline.pdf.

13. Apatira L, Boyd EA, Malvar G, Evans LR, Luce JM, Lo B, White DB: Hope, truth, and preparing for death: perspectives of surrogate decision makers. Ann Intern Med 2008; 149:861-868.

14. Glare PA, Sinclair CT: Palliative medicine review: prognostication. J Palliat Med 2008;11:84-103.

15. Back AL, Arnold RM: Discussing prognosis: "How much do you want to know?" Talking to patients who do not want information or who are ambivalent. J Clin Oncol 2006:24: 4214-4217.

16. Grimmer K, Moss J, Falco J: Experiences of elderly patients regarding independent community living after discharge from hospital: a longitudinal study. Int J Qual Health Care 2004;16:465-472.

17. Bee PE, Barnes P, Luker KA: A systematic review of informal caregivers' needs in providing home-based end-of-life care to people with cancer. J Clin Nurs. 2009;18:1379-1393.

18. Kripalani S, LeFevre F, Phillips CO: Deficits in communication and information transfer between hospital-based and primary care physicians: implications for patient safety and continuity of care. JAMA 2007;297:831-841.

19. Meier DE, Beresford L: Palliative care's challenge: facilitating transitions of care. J Palliat Med 2008;11:416-421.

20. Boling PA: Care transitions and home health care. Clin Geriatr Med 2009;25:135-148.

21. Teno JM, Clarridge BR, Casey V, Welch LC, Wetle T, Shield $\mathrm{R}$, Mor V: Family perspectives on end-of-life care at the last place of care. JAMA 2004;291;88-93.

22. Coleman EA, Parry C, Chalmers S, Min SJ: The care transitions intervention: results of a randomized controlled trial. Arch Int Med 2006;166:1822-1828.

23. Naylor MD, Brooten D, Campbell R: Comprehensive discharge planning and home follow-up of hospitalized elders: a randomized clinical trial. JAMA 1999;281:613-20.

24. Jack BW, Chetty VK, Anthony D, Greenwald JL, Sanchez GM, Johnson AE, Forsythe SR, O'Donnell JK, PaascheOrlow MK, Manasseh C, Martin S, Culpepper L: A reengineered hospital discharge program to decrease rehospitalization: a randomized trial. Ann Intern Med 2009; 150:178-187.

25. Phillips CO, Wright SM, Kern DE, Singa RM, Shepperd $S$, Rubin HR: Comprehensive discharge planning with postdischarge support for older patients with congestive heart failure: a meta-analysis. JAMA 2004;291:1358-1367.

Address correspondence to: Erik K. Fromme, M.D. Division of Hematology and Oncology Oregon Health \& Science University 3181 SW Sam Jackson Park Road Portland, OR 97239

E-mail: frommee@ohsu.edu 
